\title{
Successful Endovascular Treatment with Covered Stent for latrogenic Vertebral Arteriovenous Fistula
} Gase Report

Takuya Okata, ${ }^{1}$ Akira Ishii, ${ }^{1}$ Nobutake Sadamasa, ${ }^{1}$ Yasutoshi Kai, ${ }^{1}$ Ryota Ishibashi, ${ }^{1}$ Mitsushige Ando, ${ }^{1}$ Makoto Saka, ${ }^{1}$ Wataru Takita, ${ }^{1}$ Haruka Miyata, ${ }^{1}$ Hidehisa Nishi, ${ }^{1}$ Kazutaka Sonoda, ${ }^{1}$ Junpei Koge, ${ }^{1}$ Koichiro Futatsuya, ${ }^{2}$ and Izumi Nagata ${ }^{1}$

Objective: We discuss the details of a case in which iatrogenic vertebral arteriovenous fistula (VAVF) was successfully treated with a covered stent.

Case Presentation: A 62-year-old man presented with tinnitus after catheterization via the internal jugular vein and subsequently developed iatrogenic VAVF. Under general anesthesia, stent-assisted coil embolization was attempted via the right femoral artery but was unsuccessful. Therefore, a covered stent (Fluency $8 \times 40 \mathrm{~mm}$; C. R. Bard, Inc., Tempe, AZ, USA) was deployed, resulting in a marked decrease in shunt flow. The VAVF was obliterated 3.4 months after the procedure without postoperative complications.

Conclusion: Endovascular treatment using a covered stent can be an option for iatrogenic VAVF.

Keywords > vertebral arteriovenous fistula, endovascular treatment, covered stent, iatrogenic, pseudoaneurysm

\section{Introduction}

Vertebral arteriovenous fistula (VAVF) is a rare condition describing abnormal shunts between the extracranial vertebral artery and vertebral vein and adjacent veins. The most common cause is trauma, including iatrogenic trauma during catheterization via the internal jugular vein and during orthopedic surgery such as upper cervical spine fixation. We describe a case of iatrogenic VAVF caused by catheterization via the internal jugular vein, successfully treated by covered stent following unsuccessful attempted coil embolization.

${ }^{1}$ Department of Neurosurgery, Stroke Center, Kokura Memorial Hospital, Kitakyushu, Fukuoka, Japan

${ }^{2}$ Department of Radiology, University of Occupational and Environmental Health, Kitakyushu, Fukuoka, Japan

Received: January 18, 2017; Accepted: July 25, 2017 Corresponding author: Takuya Okata. Department of Neurosurgery, Stroke Center, Kokura Memorial Hospital, 3-2-1 Asano, Kokurakita-ku, Kitakyushu, Fukuoka 807-8556, Japan Email: taoka302@yahoo.co.jp

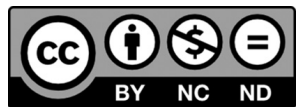

This work is licensed under a Creative Commons Attribution-NonCommercialNoDerivatives International License.

(C)2018 The Japanese Society for Neuroendovascular Therapy

\section{Case Presentation}

A 62-year-old man with a history of hypertension and dyslipidemia previously underwent catheter ablation for atrial fibrillation. He had a complaint of right-sided tinnitus approximately 30 days after the procedure and consulted a local physician 37 days after the procedure. He was referred to our department 38 days after the procedure because a vascular murmur was heard in his neck.

No neurologic abnormalities were seen on his first visit. Electrocardiography showed normal sinus rhythm, and chest radiography and transthoracic echocardiography showed no abnormalities. Carotid ultrasonography demonstrated a flap in the right vertebral artery and pulsatile vertebral vein located posterior to the vertebral artery.

Head magnetic resonance imaging and MRA showed no abnormalities (Fig. 1A). Neck MRA identified veins near the extracranial right vertebral artery (Fig. 1B). Right vertebral arterial angiography revealed arterial dissection in the $\mathrm{V} 1$ segment, a shunt between the right vertebral artery and right vertebral vein via a pseudoaneurysm, and reflux from the emissary radicular vein to the epidural venous plexus (Figs. 2 and 3). Right internal carotid arterial angiography confirmed that the right vertebral vein was contributing to normal venous drainage. Left vertebral arterial 

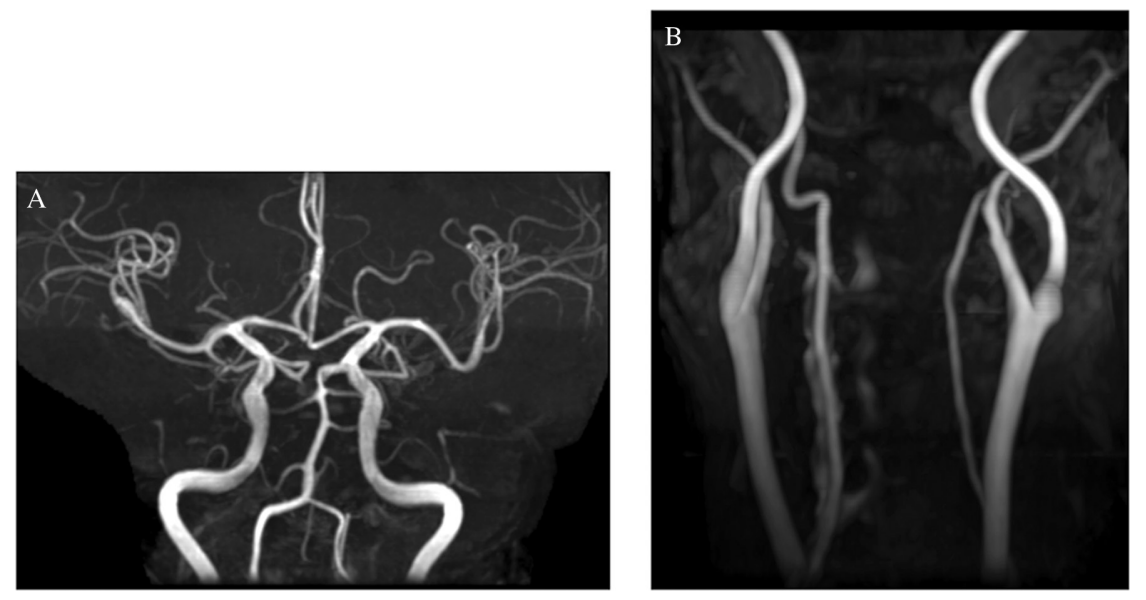

Fig. 1 (A) Head MRA showing hypoplastic left vertebral artery. (B) Neck MRA showing abnormal veins around right vertebral artery.

angiography showed right vertebral arterial dominance and no steal flow into the fistula. The patient requested treatment because of the severe pulsatile tinnitus, and endovascular treatment was performed 58 days after catheter ablation.

\section{Therapeutic strategy}

Considering his age and the right vertebral arterial dominance, occlusion of the fistula was preferable to parent artery occlusion considering the future risk of ischemic stroke. The fistula was judged to be located in a pseudoaneurysm formed on the side of a false lumen in the V1 segment (C7 level), and we chose selective transarterial/ transvenous embolization. Prior to coil embolization, we decided to perform stenting of the dissected area to reduce the shunt flow and prevent coil migration into the parent artery.

\section{Endovascular procedure}

One week before the procedure, warfarin for atrial fibrillation was replaced with continuous intravenous unfractionated heparin (10,000 units/day) with added acetylsalicylic acid (100 mg/day). Under general anesthesia, a 9F Optimo (Tokai Medical Products, Aichi, Japan) guiding catheter was placed in the right subclavian artery via the right femoral artery, and a 5F Envoy (Cordis, Johnson \& Johnson, Fremont, CA, USA) catheter was guided into the right vertebral vein via the right femoral vein. From the guiding catheter, a Carotid GuardWire (CGW) (Medtronic, Minneapolis, MN, USA) was navigated to a point sufficiently distal to the dissected area of the right V2 segment, and separate Excelsior SL-10 (Stryker, Kalamazoo, MI, USA) microcatheters were transarterially and transvenously guided to the pseudoaneurysm (fistula site) using a CHIKAI 14 (Asahi Intecc Co., Ltd, Aichi, Japan) $200 \mathrm{~cm}$ guidewire. Under distal protection with the CGW, a Precise $6 \times$ $30 \mathrm{~mm}$ stent (Cordis, Johnson \& Johnson) was deployed; however, the shunt flow could not be reduced (Fig. 4A). We attempted to form a frame with a Galaxy helical XS $2 \times 8 \mathrm{~mm}$ coil (Codman Neuro, Johnson \& Johnson, Miami, FL, USA) transarterially, but it migrated readily to the vertebral vein because of the unexpectedly large fistula. Although transvenous embolization with a Galaxy helical XS $2 \times 8 \mathrm{~mm}$ coil was also attempted, this coil also migrated to the vertebral vein side. Based on the shape of the fistula, selective embolization was judged to be difficult, and we planned to re-navigate the Excelsior SL-10 through the vertebral artery to the vertebral vein to form an anchor in the vertebral vein. Because the Axium 3D $3 \times$ $10 \mathrm{~mm}$ coil (eV3 Endovascular, Covidien, Plymouth, MN, USA) was undersized, we changed to a Galaxy Fill $5 \times$ $15 \mathrm{~mm}$ coil (Codman Neuro). However, stable frame formation was also difficult because of proximal or distal coil migration in the vertebral vein (Fig. 4A). We then changed our strategy to occlude the fistula using a covered stent (Fluency $8.0 \times 40 \mathrm{~mm}$; C. R. Bard, Inc., Tempe, AZ, USA). The 9F sheath placed in the right femoral artery was changed to a 9F Arrow $65 \mathrm{~cm}$ sheath (Arrow International, Inc., Reading, PA, USA) through a $300 \mathrm{~cm}$ Radifocus guidewire (Terumo Corporation, Tokyo, Japan), which was guided to the right subclavian artery. Then, a Fluency $8.0 \mathrm{~mm} \times 40 \mathrm{~mm}$ stent (BARD Peripheral Vascular Inc., Murray Hill, NJ, USA) was deployed to sufficiently cover the dissected lumen. While right vertebral arterial 

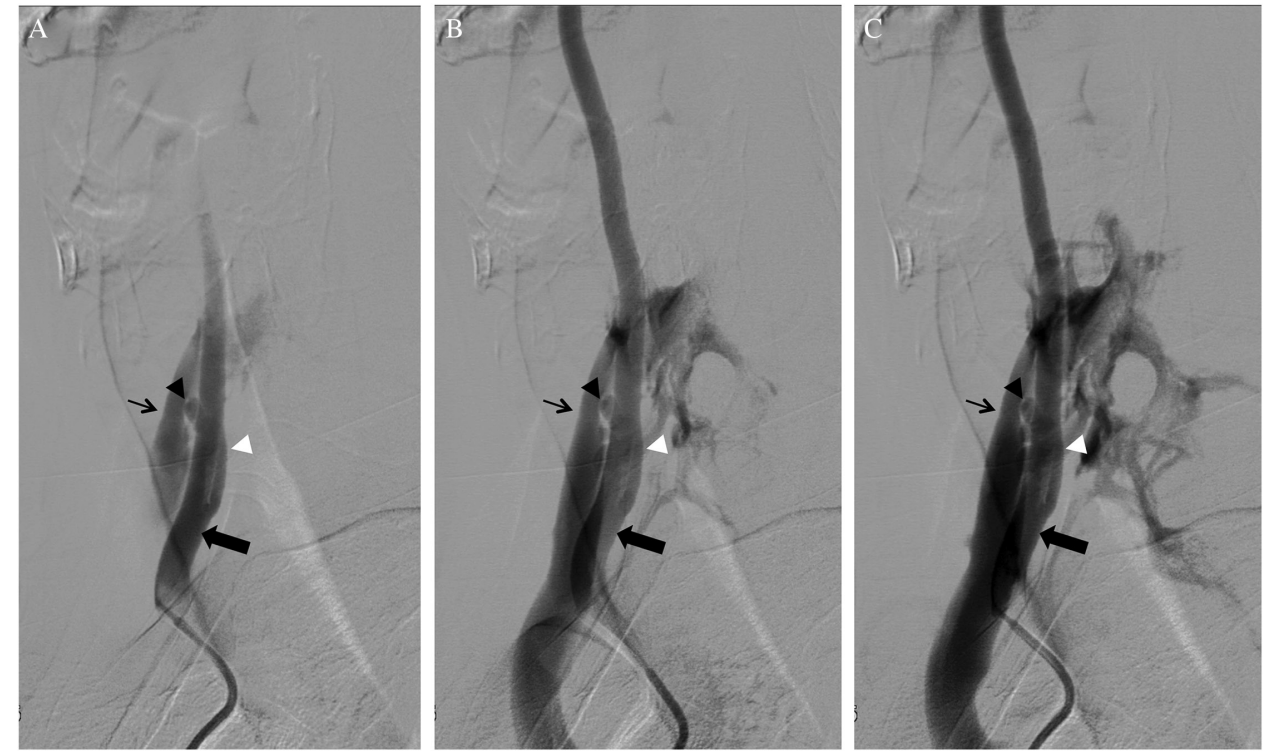

Fig. 2 Right vertebral angiograms (left anterior oblique view). (A) Early arterial phase, (B) middle arterial phase, (C) late arterial phase. A VAVF can be seen between the V1 segment (large arrow) and the vertebral vein (small arrow), accompanied with pseudoaneurysm (black arrowhead) at the site of pseudolumen (white arrowhead). VAVF: vertebral arteriovenous fistula
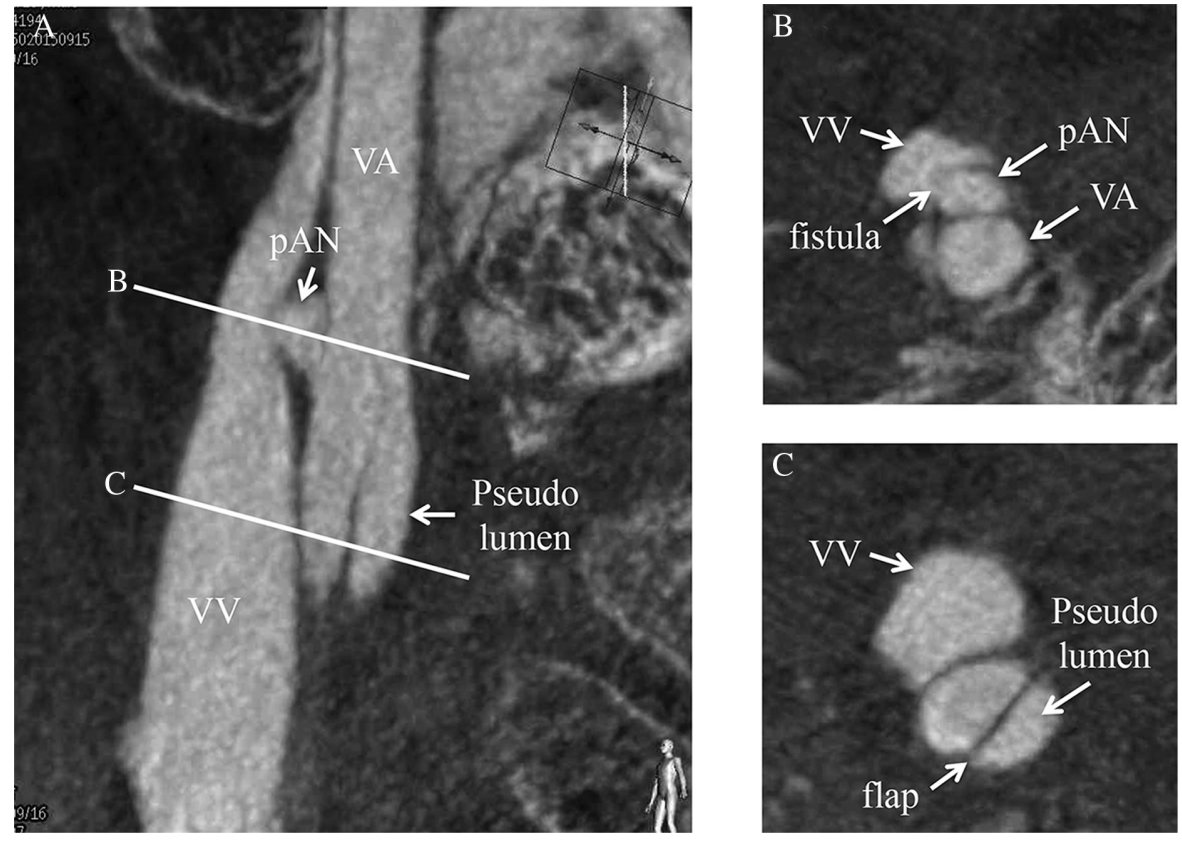

Fig. 3 (A) A reconstructed MIP image at the same projection angle as Fig. 2 demonstrating flap and pAN. (B and C) Axial images depicting location of the slice in Fig. 3A. MIP: maximum intensity profile; pAN: pseudoaneurysm; VA: vertebral artery; VV: vertebral vein

angiography showed that the right vertebral vein was slightly visualized in the delayed phase immediately after placing the Fluency stent, high-resolution cone-beam computed tomography showed adequate attachment of the stent to the vessel. We completed the procedure by judging the endoleak as type $\mathrm{IV}^{1)}$ (Fig. 5). Postoperatively, we chose antithrombotic therapy with both anticoagulant therapy for the atrial fibrillation and single antiplatelet therapy with acetylsalicylic acid for 12 months considering the potential for hemorrhagic complications. Because no perioperative complications were observed, unfractionated heparin was replaced with edoxaban $(60 \mathrm{mg} /$ day $)$ on the 


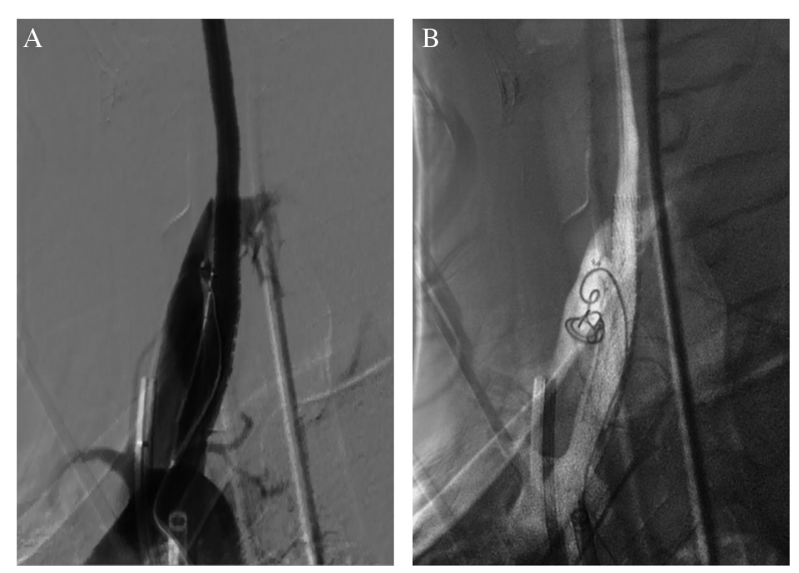

Fig. 4 (A) Angiogram immediately after deployment of Precise showed no significant change in shunt flow. (B) Angiogram showed the coil directing toward proximal part of vertebral vein by shunt flow.

third postoperative day. The shunt disappeared completely on right vertebral arterial angiography 101 days (approximately 3.4 months) after the procedure without in-stent stenosis or occlusion (Fig. 6).

\section{Discussion}

VAVF is a rare disorder in which the vertebral artery forms an abnormal shunt with the vertebral vein and adjacent veins. The most frequent clinical symptom is pulsatile tinnitus, ${ }^{2)}$ but there have also been reports of symptoms associated with vertebrobasilar ischemia (dizziness, diplopia) resulting from "steal phenomenon," which results in headache, neck pain, and cervical radicular symptoms ${ }^{3}$ from dilated epidural veins, and myelopathy associated with venous congestion. ${ }^{4}$ There is also a report of high-output heart failure in a case with a high-flow shunt and subarachnoid hemorrhage. Other than idiopathic cases, VAVF is known to be caused by trauma including iatrogenesis, atherosclerosis, angiitis, and irradiation. ${ }^{5,6)}$ Trauma accounts for $68 \%$ of the causes, and iatrogenesis is considered more frequent in Japan where stab or gunshot wounds are less frequent than in other countries., ${ }^{3,6)}$ Among iatrogenic causes of VAVF, perforation of the vertebral artery during catheterization via the internal jugular vein and orthopedic procedures such as upper cervical spine fixation have been reported. While idiopathic VAVF is usually located at the distal vertebral artery, iatrogenic VAVF is most often located at the proximal vertebral artery such as the V1 segment in a case caused by catheterization via the internal jugular vein. ${ }^{6,7)}$ Currently, no standard treatment for VAVF has been established. Although there are reports of spontaneous closure of traumatic VAVF in the acute period, spontaneous closure is difficult once a shunt has been established ${ }^{8)}$ Although VAVF has been conventionally treated surgically, reports of endovascular treatment have recently increased because of the high risk of surgical complications such as intraoperative bleeding ${ }^{4}$. If parent artery occlusion is possible, arterial embolization using a detachable balloon or coils is simplest. ${ }^{9}{ }^{10)}$ However, if preservation of the parent artery is required, selective embolization of the fistula or venous embolization is necessary. How to close the shunt as well as the approach route and embolic material should be decided based on both the shape and location of the fistula, ${ }^{11)}$ as well as the shunt flow.

If the fistula lacks sufficient space to contain the embolic material, venous embolization should be considered, and if selected, it should be noted that 1) it is necessary to clarify the shunt point and the anatomical architecture of the vertebral venous plexus, ${ }^{12)}$ and 2) a transvenous approach is difficult if the fistula is located above the V3 segment. ${ }^{12,13)}$ If the fistula is large enough for selective embolization, balloon-assisted coil embolization can be selected except for high-flow shunts, which have the risk of migration of the embolic material. However, there is a report of a patient successfully treated by coil embolization under flow control in a high-flow shunt. ${ }^{14)}$

In our patient, selective embolization was suitable considering the proximal location and sufficient space although this was a relatively high-flow shunt. There was a possibility that coil embolization could be completed using the reported flow-control technique. ${ }^{14)}$ However, we did not select embolization of the vertebral vein side for the following reasons: 1) because we observed dilatation of the vertebral vein trunk resulting from the shunt and drainage into multiple branches, it was difficult to predict the appropriate embolization area, and a considerable number of coils would have been necessary; and 2) there was a possibility of exacerbating spinal venous return if a drainage route to the venous plexus should remain. In our patient, fistula closure using a covered stent, by which radical treatment could be expected simultaneously with repair of damaged vessels, was selected as an alternative procedure.

The covered stents covered by insurance in Japan are designed for the bile duct or for the treatment of coronary artery/saphenous vein graft perforation. Currently, there are six full-covered-type covered stents available in Japan for use in the head and neck (Table 1). New-generation devices include Fluency (C. R. Bard, Inc.,) and Viabahn (W.L. Gore \& Associates, Flagstaff, AZ, USA), which was 

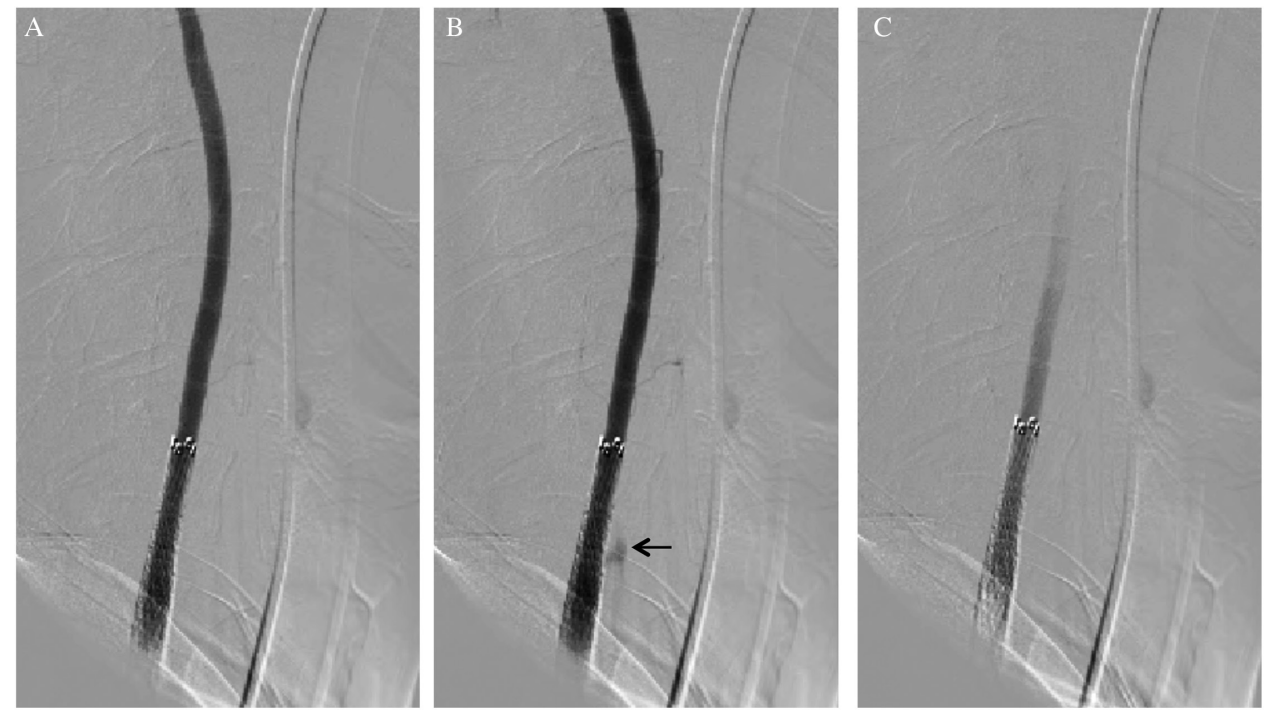

Fig. 5 (A) Early arterial phase, (B) middle arterial phase, and (C) late arterial phase. Lateral view of right vertebral artery angiogram after deployment of Fluency $8 \times 40 \mathrm{~mm}$ (C. R. Bard, Inc., Tempe, AZ, USA) demonstrated minimal residual flow through the fistula (arrow).
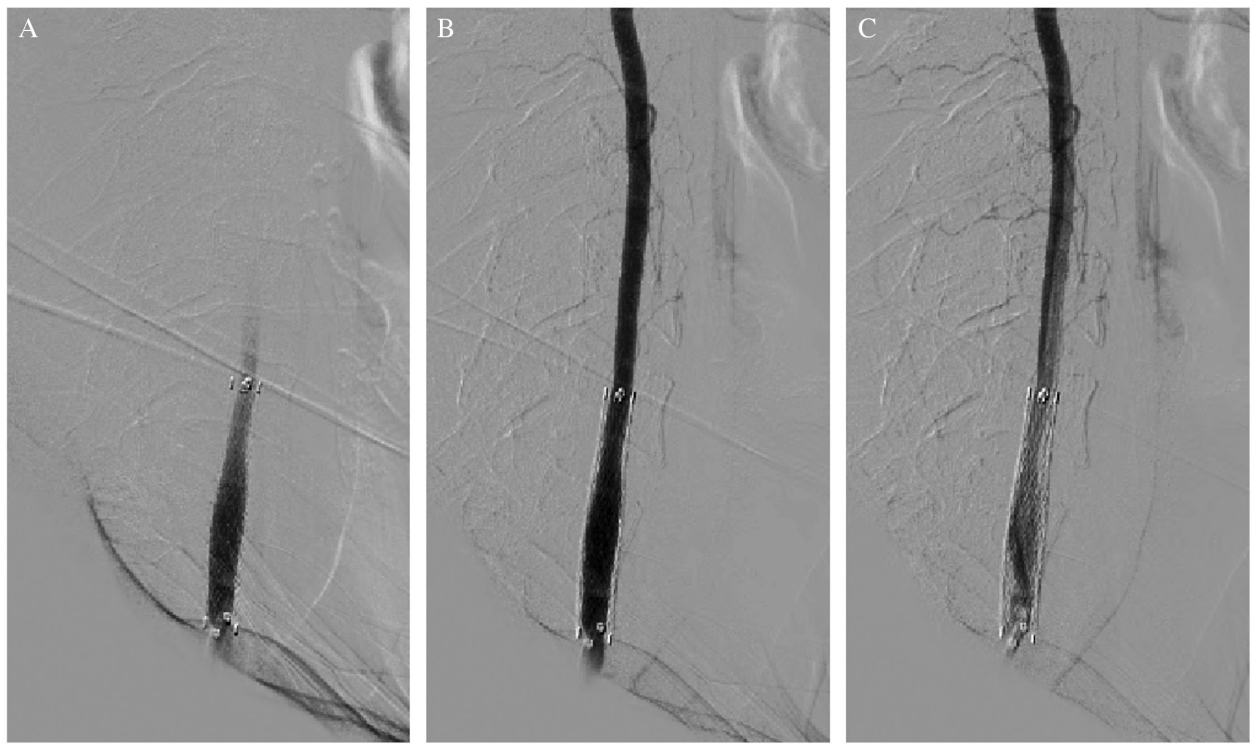

Fig. 6 (A) Early arterial phase, (B) middle arterial phase, and (C) late arterial phase. Angiogram obtained 3 months later demonstrating that the fistula has completely occluded.

introduced to the market in Japan in December 2016. The use of Viabahn for vascular injuries is now insured in Japan, and guidelines for its proper use were compiled by seven related academic societies. The guidelines require the following qualifications for operators: 1) certification or designation as an expert by the related academic societies or a physician with comparable experience; 2) experience with 100 or more cases of endovascular treatment (or experience treating under the guidance of a physician who fulfills the required number of cases); and 3) completion of a corporate-sponsored training course. The indications for using Viabahn are specified as "emergent treatment of patients who have blood leakage that is difficult to stop due to traumatic or iatrogenic injury of a thoracic, abdominal, or pelvic artery with a reference diameter of $4.0-12.0 \mathrm{~mm}$," excluding injuries to the aorta, coronary artery, brachiocephalic artery, carotid artery, pulmonary artery, and vertebral artery. The available options for the device, ranging widely in diameter (from 5 to $13 \mathrm{~mm}$ ) and length, as shown in Table 1, are characteristically richer than with other stents. 


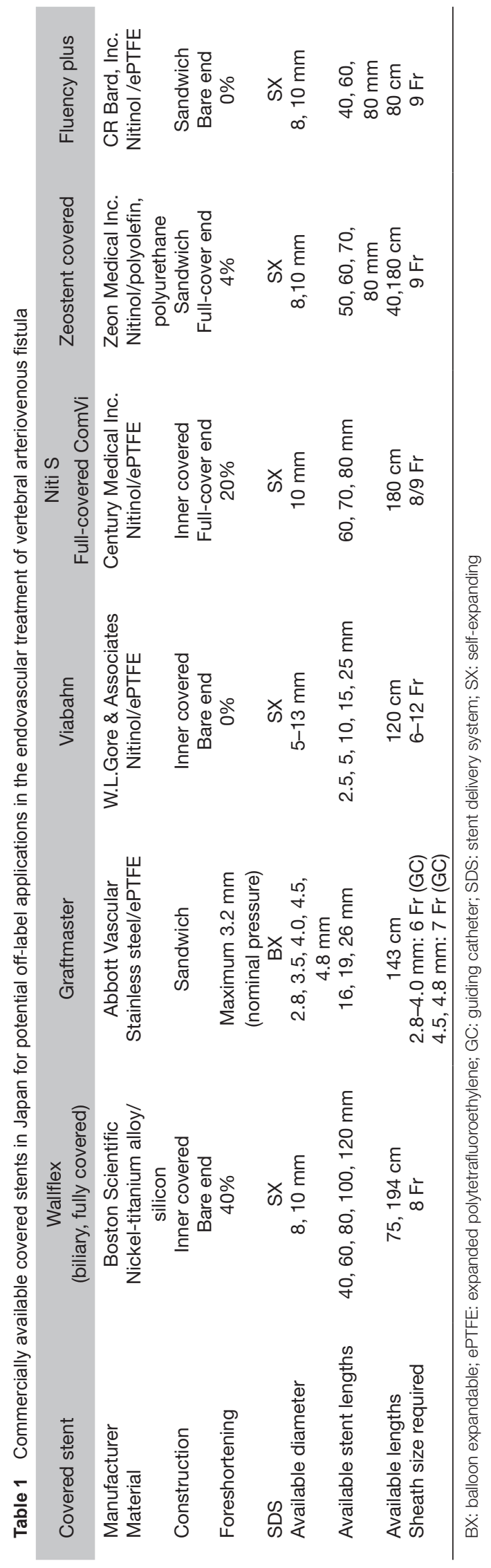

Both of these new-generation stents are self-expanding nitinol stents covered with polytetrafluoroethylene, which is reportedly less thrombogenic than polyethylene terephthalate used in previous devices. There have been several recent reports of treatment using these devices. Regardless of the stent material, structure, covering material, delivery system, or size range among devices, selection of the stent delivery-catheter length is important. The delivery catheter may be short (e.g., $80 \mathrm{~cm}$ ) or long (e.g., $180 \mathrm{~cm}$ ) although short catheters may not have sufficient length to be used for the carotid artery with distal lesions, or if the patient has a large body frame. The longer $180 \mathrm{~cm}$ Fluency catheters are available outside of Japan, but only the $80 \mathrm{~cm}$ delivery catheter is available in Japan, which we selected. Also, if an $80 \mathrm{~cm}$ delivery catheter is selected, a typical guiding sheath or guiding catheter cannot be used because these are $90 \mathrm{~cm}$ long. It must also be noted that a long sheath can also not be used; therefore, we selected a $65 \mathrm{~cm}$ guiding sheath for our patient.

Regarding the covering material, relative superiority among materials is unclear, but polytetrafluoroethylene is already in wide use as a material for artificial vessels and is considered more appropriate than polyethylene terephthalate regarding thrombogenicity.

Covered stents are self-expandable or balloon-expandable. While a balloon-expandable stent is expected to be more attachable to blood vessels, there is a risk of injury such as dissection of implanted vessels. Because high pressure is necessary for attachment of a balloon-expandable stent, caution is needed when the target vessel is already damaged. Also, as balloon-expandable delivery systems lack flexibility, a self-expandable stent is more appropriate if the target vessel is movable.

Advantages of endovascular treatment using a covered stent for VAVF are as follows: 1) the parent artery can be preserved and 2) the risk of exacerbation of radicular symptoms resulting from migration of the embolic material and a mass effect associated with coil embolization can be avoided. Disadvantages include the following: 1) a generally large size, rigidity, and poor trackability of the delivery system; 2) the development of an endoleak after placement; and 3) undefined long-term results. Endoleaks may affect therapeutic results if they are caused by an inadequate seal (Type I) in curved areas or when there is a gap between the vascular diameter at the stent placement site, or by graft failure (Type III) such as with stent-graft fractures and perforations, which are likely to occur in movable areas. Additional treatment is reported to be unnecessary for endoleaks 


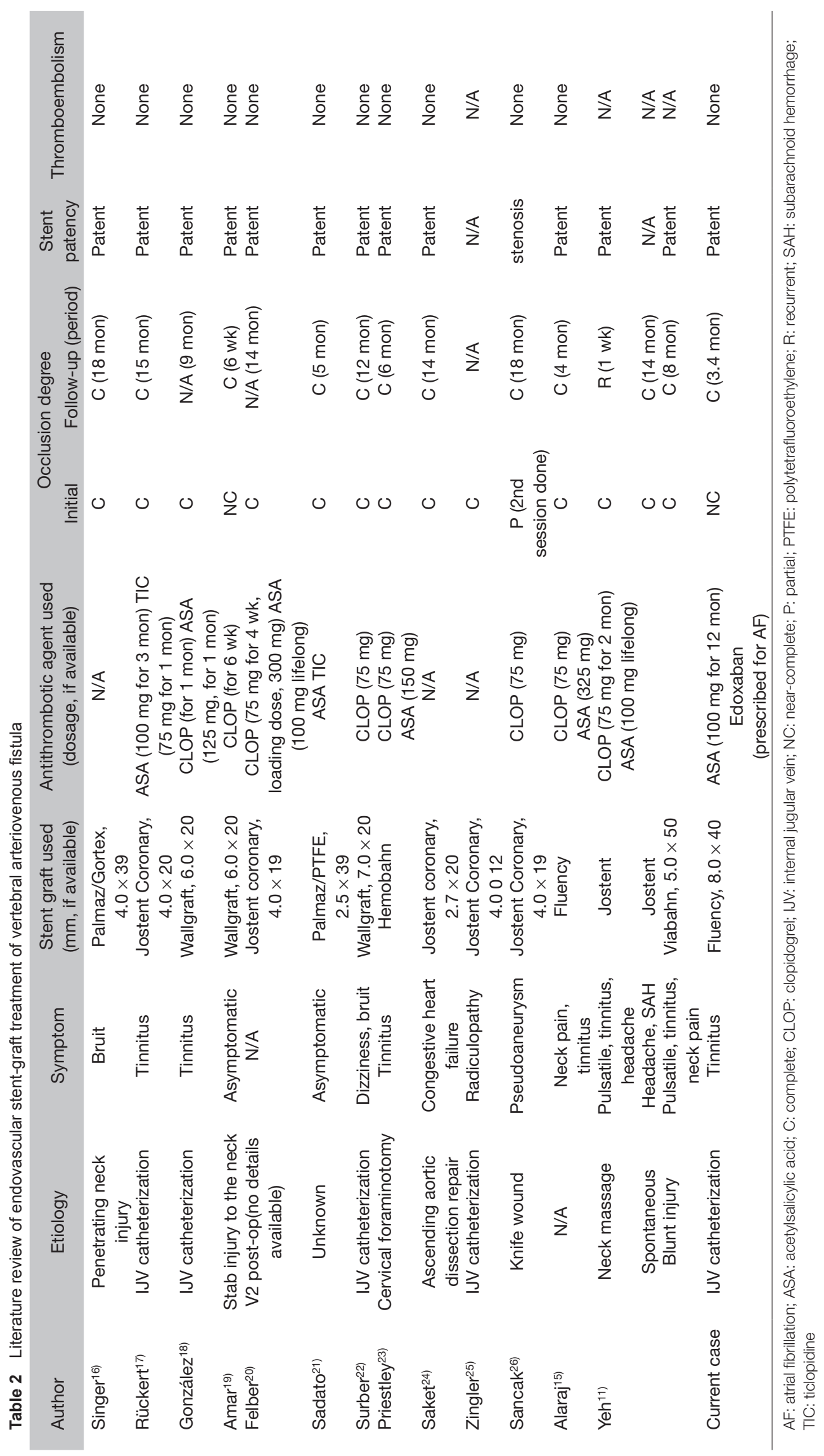


caused by stent-graft porosity (Type IV). ${ }^{1)}$ In our patient, a slight endoleak was observed immediately after treatment, but attachment to the vessel was satisfactory, and no clear graft failure was observed on high-resolution cone-beam computed tomography. The advance placement of a Precise covered stent may have some effect on endoleak development. The condition in our patient was judged to be type IV and was confirmed as completely obliterated after continued follow-up of approximately 3.4 months.

Regarding the results of endovascular treatment in the extracranial carotid artery and the vertebral artery using a covered stent, Alaraj et al. reported a systematic review of 150 cases (164 treatments). ${ }^{15)}$ By the disease, they consisted of 81 cases of pseudoaneurysm, 27 cases of carotid blowout syndrome, 23 cases of arteriovenous fistula, and 19 others. The success rate for endovascular treatment was high at $98.2 \%$ and additional treatment was performed in only one patient who developed endoleak, indicating the usefulness of the treatment. When disease is limited to VAVF, there have been 13 reports including 15 patients undergoing endovascular treatment, according to our review (Table 2). ${ }^{11,15-26)}$ Complete occlusion was achieved immediately after the procedure or at the final follow-up in 14 patients, and the procedure is considered useful in the short term. The prevalence of complications over the longterm following endovascular treatment using a covered stent in the extracranial carotid artery/vertebral artery region is reportedly $9.1 \%$ (15/164 cases), and complications included ischemic stroke in $1.2 \%$ (two cases), transient ischemic attack in $3.6 \%$ (six cases), and acute arterial dissection in $1.8 \%$ (three cases) of patients. ${ }^{15)}$ Regarding the patency rate, restenosis was observed in $2.8 \%$ (three cases), and stent occlusion was observed in $8.3 \%$ (nine cases) of the 97 cases followed-up by cerebral angiography (median follow-up period: 6 months [range, 4-17.8 months]). ${ }^{15)}$ This patency rate, calculated by including all cases of covered stent placement in the extracranial carotid and vertebral artery, is higher than the patency rate for typical stents. Evaluating the indications for treatment using a covered stent must be considered with stent occlusion in the chronic period in mind.

Optimal regimens or durations of antithrombotic therapy in the perioperative and remote periods are unclear. Previously reported antithrombotic therapies vary from acetylsalicylic acid alone to dual-antiplatelet therapy (DAPT) using acetylsalicylic acid and clopidogrel or the combination of acetylsalicylic acid and warfarin; their durations also vary from 1 month to lifetime. A standard regimen of antithrombotic treatment for carotid artery stenting is DAPT for at least 1 month with lifetime monoantiplatelet therapy. The review of Alaraj et al. ${ }^{15)}$ included a large number of patients and showed a high long-term patency rate after treatment with a covered stent; therefore, long-term DAPT, or at least one antiplatelet agent if an anticoagulant is used, is considered necessary. In our patient, because the concomitant use of an anticoagulant was necessary, we prescribed monoantiplatelet therapy; however, Alaraj et al. adopted 6 month DAPT combined with lifetime monoantiplatelet therapy based on reported evidence for carotid artery stenting. ${ }^{15)}$ Gaynor et al. also reported no ischemic complications in any of their 15 patients with carotid blowout syndrome treated using a covered stent (Viabahn). ${ }^{27)}$ Given that all patients in the report were administered two antiplatelet drugs for at least 6 weeks, DAPT may be an important factor in preventing covered stent occlusion.

\section{Conclusion}

We report a case of iatrogenic VAVF successfully treated by an endovascular procedure using a covered stent. Endovascular treatment using a covered stent is considered an option worth considering for iatrogenic VAVF.

\section{Disclosure Statement}

There are no conflicts of interest to disclose regarding this paper.

\section{References}

1) Stavropoulos SW, Charagundla SR: Imaging techniques for detection and management of endoleaks after endovascular aortic aneurysm repair. Radiology 2007; 243: 641-655.

2) Beaujeux RL, Reizine DC, Casasco A, et al: Endovascular treatment of vertebral arteriovenous fistula. Radiology 1992; 183: 361-367.

3) Gobin YP, Duckwiler GR, Viñuela F: Direct arteriovenous fistulas (carotid-cavernous and vertebral-venous). Diagnosis and intervention. Neuroimaging Clin N Am 1998; 8: 425-443.

4) Kataoka H, Miyamoto S, Nagata I, et al: Venous congestion is a major cause of neurological deterioration in spinal arteriovenous malformations. Neurosurgery 2001; 48: 1224-1229; discussion 1229-1230.

5) Halbach VV, Higashida RT, Hieshima GB: Treatment of vertebral arteriovenous fistulas. AJR Am J Roentgenol 1988; 150: 405-412. 
6) Vinchon M, Laurian C, George B, et al: Vertebral arteriovenous fistulas: a study of 49 cases and review of the literature. Cardiovasc Surg 1994; 2: 359-369.

7) Leape LL, Palacios E: Acute traumatic vertebral arteriovenous fistula. Ann Surg 1971; 174: 908-910.

8) Fukao S, Hashimoto N, Kazekawa K, et al: Percutaneous transvenous embolisation of iatrogenic vertebral arteriovenous fistula. Neuroradiology 1995; 37: 400-402.

9) Herrera DA, Vargas SA, Dublin AB: Endovascular treatment of traumatic injuries of the vertebral artery. AJNR Am J Neuroradiol 2008; 29: 1585-1589.

10) Debrun G, Legre J, Kasbarian M, et al: Endovascular occlusion of vertebral fistulae by detachable balloons with conservation of the vertebral blood flow. Radiology 1979; 130: 141-147.

11) Yeh $\mathrm{CH}$, Chen $\mathrm{YL}, \mathrm{Wu} \mathrm{YM}$, et al: Anatomically based approach for endovascular treatment of vertebrovertebral arteriovenous fistula. Interv Neuroradiol 2014; 20: 766-773.

12) Kai Y, Hamada JI, Mizuno T, et al: Transvenous embolization for vertebral arteriovenous fistula: report of two cases and technical notes. Acta Neurochir (Wien) 2001; 143: 125-8.

13) Miyachi S: [No kekkannai Chiryo Heihosho]. Medicus Shuppan, Osaka, Japan, 2015, 267. (in Japanese)

14) Takegami $T$, Imai $K$, Umezawa $K$, et al: [Endovascular trapping using a tandem balloon technique for a spontaneous vertebrovertebral fistula associated with neurofibromatosis type 1]. No Shinkei Geka 2012; 40: 705-709. (in Japanese)

15) Alaraj A, Wallace A, Amin-Hanjani S, et al: Endovascular implantation of covered stents in the extracranial carotid and vertebral arteries: case series and review of the literature. Surg Neurol Int 2011; 2: 67.

16) Singer RJ, Dake MD, Norbash A, et al: Covered stent placement for neurovascular disease. AJNR Am J Neuroradiol 1997; 18: 507-509.
17) Rückert RI, Rutsch W, Filimonow S, et al: Successful stent-graft repair of a vertebrojugular arteriovenous fistula. J Endovasc Ther 2001; 8: 495-500.

18) González A, Mayol A, Gil-Peralta A, et al: Endovascular stent-graft treatment of an iatrogenic vertebral arteriovenous fistula. Neuroradiology 2001; 43: 784-786.

19) Amar AP, Teitelbaum GP, Giannotta SL, et al: Covered stent-graft repair of the brachiocephalic arteries: technical note. Neurosurgery 2002; 51: 247-252; discussion 252-253.

20) Felber S, Henkes H, Weber W, et al: Treatment of extracranial and intracranial aneurysms and arteriovenous fistulae using stent grafts. Neurosurgery 2004; 55: 631-638; discussion 638-639.

21) Sadato A, Satow T, Ishii A, et al: Large vertebral arteriovenous fistula treated with stent-grafts-case report. Neurol Med Chir (Tokyo) 2003; 43: 250-254.

22) Surber R, Werner GS, Cohnert TU, et al: Recurrent vertebral arteriovenous fistula after surgical repair: treatment with a self-expanding stent-graft. J Endovasc Ther 2003; 10: 49-53.

23) Priestley R, Bray P, Bray A, et al: Iatrogenic vertebral arteriovenous fistula treated with a hemobahn stent-graft. J Endovasc Ther 2003; 10: 657-663.

24) Saket RR, Razavi MK, Sze DY, et al: Stent-graft treatment of extracranial carotid and vertebral arterial lesions. $J$ Vasc Interv Radiol 2004; 15: 1151-1156.

25) Zingler VC, Strupp M, Brandt $T$, et al: Stent grafting resolved brachial plexus neuropathy due to cervical arteriovenous fistula. Eur Neurol 2004; 52: 250-251.

26) Sancak T, Bilgic S, Ustuner E: Endovascular stent-graft treatment of a traumatic vertebral artery pseudoaneurysm and vertebrojugular fistula. Korean J Radiol 2008; 9: S68-S72.

27) Gaynor BG, Haussen DC, Ambekar S, et al: Covered stents for the prevention and treatment of carotid blowout syndrome. Neurosurgery 2015; 77: 164-167. 